\title{
La visión de Leibniz sobre el producto infinito de Wallis*
}

\author{
Leibniz's vision on Wallis' infinite product
}

\author{
Federico Raffo Quintana**
}

\begin{abstract}
Resumen: En este trabajo examinaré de qué manera Leibniz consideró el producto infinito de Wallis para la cuadratura del círculo. En particular, mostraré que Leibniz concibió que el resultado de Wallis no es equivalente al suyo, pues de la infinitización del producto del matemático británico, según la lectura del de Leipzig, se sigue un absurdo. De esta manera, se justificaría la concepción de Leibniz de que su propuesta de una cuadratura aritmética exacta del círculo no tiene precedentes.
\end{abstract}

Palabras clave: producto infinito, serie infinita, cuadratura del círculo, Leibniz, Wallis

Abstract: In this paper I will examine Leibniz's view on Wallis' infinite product for the quadrature of the circle. I will particularly show that Leibniz conceived that Wallis' result is not equivalent to his own one, since, according to Leibniz, the infinitization of the product of the British mathematician implies an absurd. Thus, Leibniz's conception, that his own proposal of an exact arithmetical quadrature of the circle has no precedent, would be justified.

Keywords: infinite product, infinite series, quadrature of the circle, Leibniz, Wallis

* Este trabajo fue realizado en el marco de los proyectos "La Ciencia General de Leibniz como fundamentación de las ciencias: lógica, ontología y filosofía natural" (ANPCyT PICT-2017-0506) y "Resultados de imposibilidad en geometría: perspectivas históricas y semánticas" (ANPCyT PICT-2017-0443).

** Doctor en Filosofía por la Universidad Nacional de La Plata (2017). Se desempeña como docente en la Facultad de Filosofía y Letras de la Universidad Católica Argentina. Su ámbito principal de investigación es la concepción del infinito de Leibniz. Entre sus publicaciones más recientes, se destacan Continuo e Infinito en el pensamiento leibniziano de juventud (Comares, 2019); "Leibniz on the requisites of an exact arithmetical quadrature", Studies in History and Philosopby of Science, 67, 2018, pp. 65-73 y "Leibniz in Paris: a discussion concerning the infinite number of all units" (en coautoría con Oscar Esquisabel), Revista Portuguesa de Filosofía, 73/3-4, 2017, pp. 1319-1342. Dirección electrónica: federq@gmail.com 


\section{Introducción}

Los tratamientos de Wallis y Leibniz sobre el célebre problema de la cuadratura del círculo tienen características similares. En buena medida, estas similitudes se deben a que los métodos que ambos autores propusieron son aportes puntuales dentro del campo de la matemática infinita. El desarrollo de esta rama de la matemática en el siglo XVII tuvo, en buena medida, el objetivo de buscar resolver este y otros clásicos problemas geométricos, para los cuales el mero empleo de regla y compás no era suficiente. Esto derivó, entre otras cosas, en el desarrollo de una gran variedad de 'métodos de los indivisibles'. El nombre que agrupa a estos métodos se debe a la importancia que tuvo la Geometria indivisibilibus continuorum quadam nova ratione promota, obra de B. Cavalieri publicada en 1635. La obra de Cavalieri fue, de alguna manera, un trabajo fundacional, en el sentido de que la mayoría de los matemáticos de las décadas siguientes que consideraron los problemas de cuadraturas partieron de ella. La geometría de los indivisibles se convirtió, así, en una tradición, que, como cualquier otra, alcanzó nuevas expresiones con el paso del tiempo. Este es el caso, por ejemplo, de los métodos infinitesimales desarrollados por Wallis y por Leibniz. Wallis destacó que su método comienza donde Cavalieri dejó el suyo, lo que queda plasmado en la correlación que hay entre los títulos de las obras y entre los trabajos mismos de ambos autores: "en efecto, así como la de él era la Geometría de los Indivisibles, Yo dispuse nombrar a nuestro método Aritmética de los Infinitos". ${ }^{1}$ Leibniz, por su parte, se refiere a su propio método infinitesimal como 'método de los indivisibles', pues, a pesar de que no emplea indivisibles, se inscribe en la tradición de la geometría que parte de Arquímedes y continúa con Cavalieri. ${ }^{2}$ Por ello, señala que “(...) cuando hablo de la Geometría de los indivisibles, pienso algo mucho más amplio que la

\footnotetext{
${ }^{1}$ Wallis, John, Arithmetica infinitorum, Oxford, 1656, p. Dedicatio, s/n. " ut enim ille suam, Geometriam Indivisibilium, ita Ego methodum nostram, Arithmeticam Infinitorum, nominandam duxi". Salvo que se indique otra cosa, todas las traducciones son mías.

${ }^{2}$ Leibniz, G. W., Sämtliche Schriften und Briefe (edición de la Academia de Ciencias de Berlín), Berlín (antes: Darmstadt; Leipzig), Walter de Gruyter Verlag (antes: Otto Reichl Verlag; Akademie-Verlag), 1923 y ss., serie VII, volumen 6, p. 521. De aquí en más será citado como A, seguido de la serie (en números romanos), del volumen (en números arábigos) y del número de página. Por ejemplo: A VII 6, 521.
} 
Cavaleriana, que me parece que no es sino una porción mediocre de la Arquimediana". 3

Recordemos, ante todo, que Cavalieri intentó proveer un procedimiento para hallar la razón entre dos figuras planas dadas que, por eso mismo, se apoya fuertemente en la teoría de las proporciones. Esto queda claramente de manifiesto en uno de los teoremas fundamentales del método, de acuerdo con el cual dos figuras planas tienen entre sí la misma razón que la que hay entre la colección de líneas (es decir, los 'indivisibles') de dichas figuras. ${ }^{4}$ En este sentido, supuestas dos figuras planas, $A$ y $B$, y supuestas también las colecciones de líneas de dichas figuras (obtenidas mediante un procedimiento que no analizaremos en esta ocasión), ${ }^{5}$ tenemos que $\frac{A}{B}: \frac{\text { Colección de líneas de } A}{\text { Colección de líneas de } B}$. De esta manera, si las líneas correspondientes a cada figura, pensadas siempre en una correspondencia biunívoca, estuvieran en una misma razón, entonces las dos figuras serían también magnitudes proporcionales de acuerdo con esa misma razón. ${ }^{6}$

Ahora bien, los métodos de Wallis y de Leibniz forman parte de un mismo subgrupo que se incluye en la tradición de la geometría de los indivisibles, que se caracteriza por recurrir a la aritmética. En este sentido, tanto Wallis en su Arithmetica infinitorum de 1656 como Leibniz en su De quadratura arithmetica circuli ellipseos et byperbolae (en adelante, DQA) de 1676, buscaron obtener una cuadratura del círculo mediante números o series de números. Para ambos matemáticos, esto significó, fundamentalmente, determinar la proporción que hay, por un lado, entre un círculo y un cuadrado, y, por otro, entre dos valores expresados aritméticamente. Así, por ejemplo, en la

3 A VII 6, 498. "Porro cum de Geometria indivisibilium loquor longe aliquid Cavaleriana amplius intelligo, quae mihi non videtur esse nisi portio mediocris Archimedeae".

${ }^{4}$ Cavalieri, Bonaventura, Geometria indivisibilibus continuorum quadam nova ratione promota, Boloña, 1653, pp. 113-115.

5 Sobre esto, véase Andersen, Kirsti, "Cavalieri’s Method of Indivisibles", Archive for History of Exact Sciences, 31/4, 1985, pp. 291-367; Andersen, Kirsti, "The Method of the Indivisibles: Changing Understandings", Studia Leibnitiana, Sonderheft 14, 1986, pp. 14 25; y Andersen, Kirsti, “Cavalieri’s Indivisibles”, en Jullien, Vincent (ed.), SeventeenthCentury Indivisibles Revisited, Dordrecht, Birkhäuser, 2015, pp. 31-55.

${ }^{6}$ Cavalieri, Geometria indivisibilibus continuorum, p. 115. 
proposición 190 de la Arithmetica infinitorum, Wallis señaló: "Y por lo tanto, el círculo es al cuadrado del diámetro como 1 a $m$ : $1 \mid \frac{3}{2},{ }^{7},{ }^{7}$ expresión con la que se refiere a un número (que más adelante llamaremos 'número ロ') al que nos aproximamos sucesivamente a medida que extendemos el producto infinito $\frac{3 \times 3 \times 5 \times 5 \times 7 \times 7 \text { etc. }}{2 \times 4 \times 4 \times 6 \times 6 \times 8 \text { etc. }}$, mientras que Leibniz, en la proposición XXXII de DQA, indicó: "Un círculo es al Cuadrado circunscripto (...) como $\frac{1}{1}-\frac{1}{3}+\frac{1}{5}-\frac{1}{7}+$ $\frac{1}{9}-\frac{1}{11}$ etc. a la unidad". ${ }^{8}$ Ambos resultados son muy relevantes para el problema de la cuadratura del círculo, pues el producto infinito de Wallis se iguala a $\frac{4}{\pi}$, mientras que la serie de Leibniz es igual a $\frac{\pi}{4}$, por lo que podríamos decir que son resultados equivalentes.

Estas similitudes entre sus métodos no son casuales. En buena medida, son una consecuencia del impacto que los trabajos del matemático británico generaron en Leibniz, en especial durante el período que pasó en París. Entre 1672 y 1673, Leibniz leyó varios artículos de Wallis publicados en Philosophical Transactions, así como también el libro recién salido a la luz Mechanica sive de motu tractatus geometricus (1670), que también contiene buena parte de los principales resultados matemáticos que Wallis había obtenido en los años previos. Naturalmente, esto explica que las referencias a Wallis en los escritos de Leibniz de este período se den, especialmente, en el dominio de la matemática infinita. A esto hay que sumarle que, en los años siguientes, Leibniz leyó con detenimiento la Arithmetica infinitorum, publicada casi veinte años antes. Ahora bien, la valoración que Leibniz hizo de los trabajos de Wallis es ambigua. Como señala Probst, "Leibniz reconoció los logros de Wallis, pero se mantuvo crítico con respecto a la falta de demostraciones rigurosas en los textos de Wallis". ${ }^{9}$

${ }^{7}$ Wallis, Arithmetica infinitorum, p. 175. "Et propterea circulus est ad quadratum diametri, ut 1 ad $m: 1 \mid \frac{3}{2}$,"

8 A VII 6, 600. "Circulus est ad Quadratum circumscriptum (...) ut $\frac{1}{1}-\frac{1}{3}+\frac{1}{5}-\frac{1}{7}+$ $\frac{1}{9}-\frac{1}{11}$ etc. ad unitatem".

9 Probst, Siegmund, "The Relation between Leibniz and Wallis: an Overview from New Sources and Studies", Quaderns d'Història de l'Enginyeria, XVI, 2018, p. 195. Los datos sobre la periodización de las lecturas de Leibniz los tomamos también de este trabajo. 
En este trabajo examinaremos una crítica puntual, vinculada, podríamos decir, con el alcance del resultado propuesto por Wallis para la cuadratura del círculo. Veremos, en pocas palabras, que, para Leibniz, el producto de Wallis para $\frac{4}{\pi}$ no contribuye en la búsqueda de una expresión exacta del círculo, dado que se seguirían absurdos si se lo presumiera infinito en acto.

Dividiremos el trabajo en cuatro partes. En la primera de ellas, reconstruiremos sintéticamente el camino que condujo a Wallis a proponer el producto infinito para la cuadratura del círculo, así como también algunos presupuestos generales de su planteo. Esto nos permitirá examinar, en las secciones siguientes, la crítica de Leibniz. En la segunda parte, presentaremos la clasificación de los tipos de cuadraturas propuesta por Leibniz, lo que dejará en evidencia el modo en que el filósofo de Leipzig estimó el resultado de Wallis para la cuadratura del círculo, a saber, como un cómputo que permite aproximarse a un valor para el círculo, aunque no provee de un resultado exacto. En siguiente lugar, mostraremos que para Leibniz el producto de Wallis no puede dar un resultado exacto, pues, supuesta su infinitización, y tras realizar ciertas operaciones algebraicas, se obtiene como resultado una cantidad infinitamente pequeña. En consecuencia, en cuarto lugar, mostraremos por qué para Leibniz una cantidad infinitamente pequeña implica absurdos.

\section{E1 producto de Wallis}

El tratamiento del problema de la cuadratura del círculo llevado a cabo por Wallis en la Arithmetica infinitorum se caracteriza por tener un claro componente diagramático. Esto se observa en el recurrente uso de tablas en las que expone números, las cuales son tenidas muy en cuenta por Wallis cuando busca una expresión que le provea la cuadratura del círculo. Este aspecto visual llevó a que Leibniz, un tiempo más adelante, considere que la aritmética de Wallis es 'figurada', a diferencia de la suya que es 'pura'. ${ }^{10}$ Wallis exhibe las sucesiones del triángulo aritmético (esto es, las que más adelante se conocerán como las sucesiones del 'triángulo de Pascal'), ${ }^{11}$ es decir, primero la de las unidades, luego

${ }^{10}$ A VII 3, 102. Si la apreciación que Leibniz hizo de su propia aritmética es correcta o no, es una cuestión que no trataremos en esta oportunidad.

${ }^{11}$ Esto se debe al tratamiento que se le concedió en Pascal, Blaise, Traité du triangle arithmétique, avec quelques autres petits traitez. sur la mesme matière, París, G. Desprez, 1665. 
la de los números naturales (o 'lados'), en tercer lugar la de los triangulares, etc. $^{12}$ Esta exposición de tablas de números es fundamental, pues Wallis sostiene que la razón que hay entre un círculo y el cuadrado de su diámetro es como la del número 1 a otro número (pero no a una serie de números, como luego sostendrá Leibniz), que es una cantidad incógnita y al que se refiere empleando el signo $\square$ (de modo que $\frac{\text { Círculo }}{\text { Cuadrado }}: \frac{1}{\square}$ ). En términos más actuales, este número corresponde a $\frac{4}{\pi} \cdot 13$

Sobre la base de estas presuposiciones, podríamos decir, de un modo muy esquemático, que el planteo de Wallis tiene, al menos, estos momentos centrales: (1) debemos ubicar, es decir, literalmente, determinar el lugar de, el número $\square$ en la tabla de números; (2) debemos examinar si es posible asignarle un número racional a dicho número; y (3), en caso de que no lo fuera, debemos ofrecer un cálculo para dar con este número. Como veremos, Wallis no podrá asignarle un número racional al número $\square \mathrm{y}$, por lo tanto, proveerá una operación que nos permite aproximarnos a él. Detengámonos sucintamente en cada uno de estos momentos.

(1) En la Arithmetica infinitorum, Wallis emplea un procedimiento que llamó 'método de interpolación' y que consiste en determinar el término medio entre dos términos sucesivos de una progresión, sea aritmética, geométrica o de un tipo mixto más complejo, de acuerdo con el modo en que la sucesión progresa. ${ }^{14}$ Así, por ejemplo, entre 1, 2, 3, 4, etc. se interponen respectivamente $\frac{3}{2}, \frac{5}{2}, \frac{7}{2}, \frac{9}{2}$, etc. $^{15}$ Wallis es consciente de que en otras sucesiones la interpolación

12 Wallis sigue la denominación que ofreció Oughtred en Clavius mathematicae (nota 16 del capítulo 18 de la primera edición de 1631, o en la nota 11 del capítulo 17 de la segunda edición de 1647).

${ }^{13}$ Esto implica, como dijimos, que el número $\square$ es el número al que se refiere con la expresión $m: 1 \mid \frac{3}{2}$ señalada antes. Esta expresión hace referencia a que el número $\square$ es el término interpolado entre 1 y $\frac{3}{2}$ en la sucesión vertical u horizontal correspondiente, según se podrá ver más abajo en la Tabla 2. La noción de 'interpolación’ será explicada a continuación.

${ }^{14}$ Wallis, Arithmetica infinitorum, p. marcada como Bb.

${ }^{15} \mathrm{Ibid}$., p. 138. Estos números son expresados en el texto y en las tablas que veremos más adelante, respectivamente, de este modo: $\frac{1}{2}, 1 \frac{1}{2}, 2 \frac{1}{2}, 3 \frac{1}{2}, 4 \frac{1}{2}$, etc. 
no es tan sencilla. Para esos casos, necesitaremos hallar primero el "carácter propio de la serie" (seriei propio charactere), es decir, el procedimiento por el que generamos todos los términos que la componen (o sea, el término general). Así, por ejemplo, entre los números triangulares, 1, 3, 6, 10, 15, etc. (esto es, de los cuales el término general es $\left.\frac{n^{2}+n}{2}\right)$, se interponen respectivamente $\frac{15}{8}$ (para $n=$ $\frac{3}{2}$, es decir, el número interpolado entre 1 y 2 ), $\frac{35}{8}$ (para $n=\frac{5}{2}$ ), $\frac{63}{8}$ (para $n=$ $\frac{7}{2}$ ), $\frac{99}{8}$ (para $n=\frac{9}{2}$ ), y así sucesivamente. ${ }^{16}$ De este modo, si disponemos en orden los números naturales y los triangulares incluyendo los términos interpolados entre ellos, tenemos:

- Números naturales: $\frac{1}{2}, 1, \frac{3}{2}, 2, \frac{5}{2}, 3, \frac{7}{2}, 4, \frac{9}{2}$, etc.

- Números triangulares: $\frac{3}{8}, 1, \frac{15}{8}, 3, \frac{35}{8}, 6, \frac{64}{8}, 10, \frac{99}{8}, 15$, etc.

Tengamos en cuenta esta tabla:

16 Ibid., p. 141. En este caso, las fracciones se expresan respectivamente $\frac{3}{8}, 1 \frac{7}{8}, 4 \frac{3}{8}, 7 \frac{7}{8}, 12 \frac{3}{8}$, etc. 


\begin{tabular}{|c|c|c|c|c|c|c|c|c|c|c|}
\hline & & 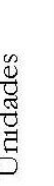 & & 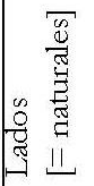 & & 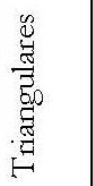 & & 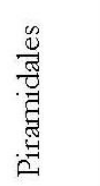 & & 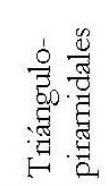 \\
\hline & $\infty$ & 1 & & $\frac{1}{2}$ & & $\frac{3}{8}$ & & $\frac{15}{48}$ & & $\frac{105}{384}$ \\
\hline \multirow[t]{2}{*}{ Unidades } & 1 & 1 & 1 & 1 & 1 & 1 & 1 & 1 & 1 & 1 \\
\hline & & 1 & 口 & $1 \frac{1}{2}$ & & $1 \frac{7}{8}$ & & $2 \frac{9}{48}$ & & $2 \frac{177}{384}$ \\
\hline \multirow[t]{2}{*}{$\begin{array}{l}\text { Lados } \\
{[=\text { naturales }]}\end{array}$} & $\frac{1}{2}$ & 1 & $1 \frac{1}{2}$ & 2 & $2 \frac{1}{2}$ & 3 & $3 \frac{1}{2}$ & 4 & $4 \frac{1}{2}$ & 5 \\
\hline & & 1 & & $2 \frac{1}{2}$ & & $4 \frac{3}{8}$ & & $6 \frac{27}{48}$ & & $9 \frac{9}{384}$ \\
\hline \multirow[t]{2}{*}{ Triangulares } & $\frac{3}{8}$ & 1 & $1 \frac{7}{8}$ & 3 & $4 \frac{3}{8}$ & 6 & $7 \frac{7}{8}$ & 10 & $12 \frac{3}{8}$ & 15 \\
\hline & & 1 & & $3 \frac{1}{2}$ & & $7 \frac{7}{8}$ & & $14 \frac{21}{48}$ & & $23 \frac{177}{384}$ \\
\hline \multirow[t]{2}{*}{ Piramidales } & $\frac{15}{48}$ & 1 & $2 \frac{9}{48}$ & 4 & $6 \frac{27}{48}$ & 10 & $14 \frac{21}{48}$ & 20 & $26 \frac{39}{48}$ & 35 \\
\hline & & 1 & & $4 \frac{1}{2}$ & & $12 \frac{3}{8}$ & & $26 \frac{39}{48}$ & & $50 \frac{105}{384}$ \\
\hline $\begin{array}{l}\text { Triángulo- } \\
\text { piramidales }\end{array}$ & $\frac{105}{384}$ & 1 & $2 \frac{177}{384}$ & 5 & $9 \frac{9}{384}$ & 15 & $23 \frac{177}{384}$ & 35 & $50 \frac{105}{384}$ & 70 \\
\hline
\end{tabular}

[Tabla 1: proposición 184 de Aritbmetica infinitorum]

Como vemos, entre los términos de algunas sucesiones, Wallis dejó espacios en blanco, que deben ser llenados de acuerdo con el método de interpolación. Además de los números del triángulo aritmético, Wallis incluyó en un casillero particular de esta tabla el número $\square$. El razonamiento que siguió 
para incluir este número es muy complejo, por lo que no lo examinaremos en detalle en esta ocasión. En pocas palabras, puede sintetizarse de este modo: ${ }^{17}$ - En la imagen, $\mathrm{R}$ es el radio del círculo, del cual $\frac{R}{\infty}=a$ es una parte infinitamente pequeña. Dibujemos infinitas perpendiculares que lleguen desde el radio al cuadrante del círculo. Dichas perpendiculares serán medias proporcionales entre segmentos del diámetro.

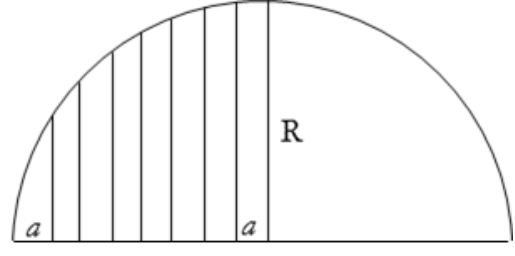

$\mathrm{R} \quad \mathrm{R}$

[Imagen 1: proposición 121 de Arithmetica infinitorum]

- Por lo tanto, la razón de la suma de todas las medias proporcionales al radio es la misma que tiene el cuadrante del círculo al cuadrado del diámetro. Y por ello, también todo el círculo al cuadrado del diámetro.

- Ahora bien, esta misma razón es la que tiene la unidad al número intermedio entre 1 y $\frac{3}{2}$ (véase proposición 168). Si a este número intermedio los llamamos $\square$, podemos decir que $\frac{\text { círculo }}{\text { cuadrado }}: \frac{1}{\square}$. Desde la proposición 121 en adelante, Wallis se dedica a investigar el modo de hallar este número.

Dado el estado actual de la tabla, en la que pocos números se han aún revelado, podemos saber que el círculo es al cuadrado del diámetro como $1 \mathrm{a}$ $\square$, esto es, al número interpuesto entre 1 y 2 en la serie de los diagonales 1,2 , $6,20,70$, etc. de la tabla, ${ }^{18}$ pero no mucho más que eso. De este modo, si contáramos con el 'carácter propio' de la serie diagonal (lo que no es el caso),

${ }^{17}$ Ibid., pp. 91-92. Para un desarrollo más detallado y sofisticado de esta cuestión, véase Malet, Antoni y Panza, Marco, "Wallis on Indivisibles", en Jullien, Vincent (ed.), Seventeenth-Century Indivisibles Revisited, especialmente pp. 334-336.

${ }^{18}$ Wallis, Arithmetica infinitorum, p. 135. 
podríamos determinar este número. Siguiendo el método de interpolación, Wallis continúa completando la tabla, hasta el punto en el que tenemos números asignados en los casilleros correspondientes a las sucesiones del triángulo aritmético, pero para las sucesiones interpoladas entre ellas (es decir, las ubicadas en las posiciones impares de la tabla), y en especial para los términos impares de dichas sucesiones, no disponemos de números racionales. Solamente conocemos el tercer término de la tercera de estas sucesiones, que es el número $\square$, tal como podemos observar en la última tabla. Por esta razón, Wallis refiere todos los números que restan completar al número $\square$. El resultado de todo esto puede observarse en la siguiente tabla:

\begin{tabular}{|c|c|c|c|c|c|c|c|c|c|c}
\hline$\infty$ & 1 & $\frac{1}{2} \square$ & $\frac{1}{2}$ & $\frac{1}{3} \square$ & $\frac{3}{8}$ & $\frac{4}{15} \square$ & $\frac{15}{48}$ & $\frac{8}{35} \square$ & $\frac{105}{384}$ & $A$ \\
\hline 1 & 1 & 1 & 1 & 1 & 1 & 1 & 1 & 1 & 1 & 1 \\
\hline$\frac{1}{2} \square$ & 1 & $\square$ & $\frac{3}{2}$ & $\frac{4}{3} \square$ & $\frac{15}{8}$ & $\frac{8}{5} \square$ & $\frac{105}{48}$ & $\frac{64}{35} \square$ & $\frac{945}{384}$ & $A \times \frac{2 l-1}{1}$ \\
\hline$\frac{1}{2}$ & 1 & $\frac{3}{2}$ & 2 & $\frac{5}{2}$ & 3 & $\frac{7}{2}$ & 4 & $\frac{9}{2}$ & 5 & $l=\frac{2 l+0}{2}$ \\
\hline$\frac{1}{3} \square$ & 1 & $\frac{4}{3} \square$ & $\frac{5}{2}$ & $\frac{8}{3} \square$ & $\frac{35}{8}$ & $\frac{64}{15} \square$ & $\frac{315}{48}$ & $\frac{128}{21} \square$ & $\frac{3465}{384}$ & $A \times \frac{4 l^{2}-1}{3}$ \\
\hline$\frac{3}{8}$ & 1 & $\frac{15}{8}$ & 3 & $\frac{35}{8}$ & 6 & $\frac{63}{8}$ & 10 & $\frac{99}{8}$ & 15 & $\frac{l^{2}+l}{2}=\frac{4 l^{2}+4 l}{8}$ \\
\hline$\frac{4}{15} \square$ & 1 & $\frac{8}{5} \square$ & $\frac{7}{2}$ & $\frac{64}{15} \square$ & $\frac{63}{8}$ & $\frac{128}{15} \square$ & $\frac{693}{48}$ & $\frac{512}{35} \square$ & $\frac{9009}{384}$ & $A \times \frac{8 l^{3}+12 l^{2}-2 l-3}{15}$ \\
\hline$\frac{15}{48}$ & 1 & $\frac{105}{48}$ & 4 & $\frac{315}{48}$ & 10 & $\frac{693}{48}$ & 20 & $\frac{1287}{48}$ & 35 & $\frac{l^{3}+3 l^{2}+2 l}{6}=\frac{8 l^{3}+24 l^{3}+16 l}{48}$ \\
\hline$\frac{8}{35} \square$ & 1 & $\frac{64}{35} \square$ & $\frac{9}{2}$ & $\frac{128}{21} \square$ & $\frac{99}{8}$ & $\frac{512}{35} \square$ & $\frac{1287}{48}$ & $\frac{1024}{35} \square$ & $\frac{19305}{384}$ & $A \times \frac{16 l^{4}+64 l^{3}+56 l^{2}-16 l-15}{105}$ \\
\hline$\frac{105}{384}$ & 1 & $\frac{945}{384}$ & 5 & $\frac{3465}{384}$ & 15 & $\frac{9009}{384}$ & 35 & $\frac{19305}{384}$ & 70 & $\frac{6 l^{4}+6 l^{3}+11 l^{2}+6 l}{24}=\frac{16 l^{4}+96 l^{3}+176 l^{2}+6 l}{384}$ \\
\hline
\end{tabular}

[Tabla 2: proposición 189 de Arithmetica infinitorum]

Para cualquiera de las columnas, ${ }^{19} A$ es el primer término (es decir, el primer número de los que están ubicados en casilleros impares) y 1, el segundo (el primero de los que están ubicados en casilleros pares). Como se observa, los siguientes se obtienen de acuerdo con las reglas halladas por Wallis. En

${ }^{19}$ Opto por describir la tabla atendiendo a las columnas, pero, como puede verse, puede también leerse por las filas. 
cualquier de ellas, l es el término correspondiente de la sucesión de los números naturales. Por ejemplo, el tercer término de la cuarta columna (es decir, $\frac{3}{2}$ ) se obtiene mediante la fórmula $A \times \frac{2 l-1}{1}$. Dado que, para la cuarta columna, $A=$ $\frac{1}{2}$, entonces $\frac{1}{2} \times \frac{(2 \times 2)-1}{1}=\frac{3}{2}$ (donde $l$ fue reemplazado por 2 por ser el término siguiente a la unidad).

(2) Como vemos, Wallis 'completó' la tabla, pero no pudo asignar un número racional para el número $\square$. Bastaría con conocer un número cualquiera de los números impares de cualquiera de las sucesiones impares, para dar con dicho número, pues en ese caso podríamos despejar el número $\square$. De aquí, Wallis concluye que el número $\square$ es un número imposible, esto es, que no podemos asignarle un número de acuerdo con las notaciones disponibles. ${ }^{20}$

(3) Por eso, Wallis hace lo que suelen hacer los aritméticos en este tipo de situaciones: supone que se da el número $\square$ (aunque de hecho no se dé) y busca determinar una manera de representarlo. Para esto, como resultado, Wallis recurre a otro tipo de cálculos, que, si bien no nos darán el número buscado, nos permitirán aproximarnos a él todo lo que queramos. En este camino, toma los términos de la tercera columna (es decir, los términos de la sucesión en la que tiene lugar en número $\square$ ) y, en orden, nombra a los impares con letras griegas y a los pares con caracteres latinos, es decir: $\alpha: \frac{1}{2} \square, \beta: \square, \gamma$ : $\frac{4}{3} \square, \delta: \frac{64}{15} \square$ (Wallis lo presenta aquí como $\frac{4 \times 6}{3 \times 5} \square$ ), etc., así como a: 1 , b: $\frac{3}{2}$, c: $\frac{15}{8}$ (que presenta como $\frac{3 \times 5}{2 \times 4}$ ), d: $\frac{105}{48}$ (o sea, $\frac{3 \times 5 \times 7}{2 \times 4 \times 6}$ ), etc. En siguiente lugar, muestra que $\frac{\alpha}{\beta}: \frac{1}{2}$, que $\frac{a}{b}: \frac{2}{3}$, y así sucesivamente: $\frac{\beta}{\gamma}: \frac{3}{4} ; \frac{b}{c}: \frac{4}{5} ; \frac{\gamma}{\delta}: \frac{5}{6} ; \frac{c}{d}: \frac{6}{7}$; etc. De allí es sencillo notar que, en consecuencia, $\frac{\beta}{\alpha}: \frac{2}{1}$, y lo mismo para los demás casos $\left(\frac{b}{a}: \frac{3}{2} ; \frac{\gamma}{\beta}: \frac{4}{3} ; \frac{c}{b}: \frac{5}{4} ; \frac{\delta}{\gamma}: \frac{6}{5} ; \frac{d}{c}: \frac{7}{6} ;\right.$ etc.). Como vemos, la proporción es decreciente. Ahora bien, dado esto, en siguiente lugar, sostiene que $\frac{\beta}{a}$ es la menor de estas

${ }^{20} \mathrm{Ibid}$, p. 174. 
dos fracciones: $21 \frac{a}{\alpha}$ y $\frac{\beta}{a}$, de modo que, por lo tanto, es menor que la raíz cuadrada de su producto: $\frac{a}{\alpha} \times \frac{\beta}{a}=\frac{\beta}{\alpha}$ (dado que $a$ es 1 ), por lo que $\frac{\beta}{a}<\sqrt{\frac{2}{1}}$. En consecuencia, $\beta$, que, recordemos, es $\square$, es $<1 \sqrt{2}$ (que Wallis también expresa $1 \sqrt{1 \frac{1}{1}}$. Por un razonamiento análogo, podemos saber que $\frac{\beta}{a}$ es, en este caso, la mayor de estas dos fracciones: $\frac{\beta}{a}$ y $\frac{\mathrm{b}}{\beta}$, de donde resulta que es mayor que la raíz cuadrada del producto de dichas fracciones. En consecuencia, $\beta>1 \sqrt{\frac{3}{2}}$ (o también $1 \sqrt{1 \frac{1}{2}}$ ). Un razonamiento análogo podemos aplicarlo para determinar con mayor precisión no ya $\frac{\beta}{a}$, sino $\frac{\gamma}{b}$. En este caso, podemos saber que es la menor de estas dos fracciones: $\frac{\mathrm{b}}{\beta} \mathrm{y} \frac{\gamma}{b}$, por lo que es $<\sqrt{\frac{4}{3}}=\sqrt{1 \frac{1}{3}}$, y es la mayor de estas dos fracciones: $\frac{\gamma}{b}$ y $\frac{c}{\gamma}$, por lo que es $>\sqrt{\frac{5}{4}}=\sqrt{1 \frac{1}{4}}$. En consecuencia, $\gamma=b \times \frac{\gamma}{b}$, esto es, $\frac{4}{3} \square$, lo que es $<\frac{3}{2} \times \sqrt{1 \frac{1}{3}}$ y $>\frac{3}{2} \times \sqrt{1 \frac{1}{4}}$. De allí podemos obtener que $\square$ es $<\frac{3 \times 3}{2 \times 4} \times \sqrt{1 \frac{1}{3}}$ y $>\frac{3 \times 3}{2 \times 4} \times \sqrt{1 \frac{1}{4}}$. Un razonamiento similar puede aplicarse para determinar $\frac{\delta}{c}$, de lo que obtendremos que $\square<$ $\frac{3 \times 3 \times 5 \times 5}{2 \times 4 \times 4 \times 6} \times \sqrt{1 \frac{1}{5}}$, pero $>\frac{3 \times 3 \times 5 \times 5}{2 \times 4 \times 4 \times 6} \times \sqrt{1 \frac{1}{6}}$. Esto ya le basta a Wallis para detectar que hay una regla para obtener sucesivamente una aproximación, tanto mayor como menor, al número $\square$ :

Y así sucesivamente todo lo que se quiera, de modo que el Numerador de la fracción surja de multiplicar continuamente los números impares 3, 5, 7,

\footnotetext{
${ }^{21}$ Tengo en cuenta la aclaración que señala Stedall en la nota 77 de su traducción del texto: Wallis, John, The Arithmetic of Infinitesimals, traducción de Jacqueline Stedall, New York, Springer, 2004, p. 165.
} 
etc. puestos dos veces [cada uno], y el Denominador, de multiplicar continuamente los números pares, 2,4 , 6, etc., también puestos dos veces, excepto el primero y el último, que se ponen una vez. Finalmente, toda esta razón o fracción producida de esta manera, se multiplica por la Raíz cuadrada de la unidad más una parte alícuota suya, que tiene como denominador el último de los números impares multiplicados continuamente, si buscamos el número mayor correspondiente, o de los pares, si [buscamos] el menor correspondiente.

$\mathrm{Y}$ [siguiendo] este procedimiento todo lo que se quiera, finalmente arribaremos a que la diferencia entre el mayor y el menor llega a ser menor que cualquiera asignada (que, por lo tanto, si se supone que la operación continúa al infinito, finalmente desaparecerá). ${ }^{22}$

Como vemos, podemos designar valores que se aproximan al número con números racionales: es mayor que 1,27, pero menor que 1,28; es mayor que 1,2732 y menor que 1,2733 ; es mayor que 1,273239 y menor que 1,273240 , y así sucesivamente, pero nunca podemos proveer un valor exacto. ${ }^{23}$

\section{La clasificación de las cuadraturas según Leibniz}

Como sabemos, en el siglo XVII, el problema de la cuadratura del círculo fue abordado por muchos autores y en una gran variedad de tratados. ${ }^{24}$ Las

22 Ibid., pp. 179-180. "Et sic deinceps quousq; libet. Ita nempe ut fractionis Numerator fiat continue multiplicando numeros impares 3, 5, 7, \&c. bis positos; Denominator vero, continue multiplicando numeros pares $2,4,6$, \&c. bis item positos, exceptis primo \& ultimo, qui semel ponuntur: Et tota deniq; ratio seu fractio, sic facta, ducatur in Radicem-quadraticam Unitatis aliquota-parte sui auctae; ea nempe quae denominatorem habet eum qui est ultimus numerorum, continue multiplicatorum, imparium, si quaramus numerum justo majorem, vel parium, si justo minorem. / Atq; hoc pacto eousq; tandem pervenietur donec majoris \& minoris differentia evadat quavis assignata minor; (quae propterea, si supponatur in infinitum continuanda operatio, tandem evaditura est)".

${ }^{23}$ Ibid., p. 176.

${ }^{24}$ Además de los trabajos de Wallis y Leibniz que ya mencionamos, se destacan, entre muchos otros, van Ceulen, Ludolph, Vanden Circkel, Delft, Jan Andriesz Boeckvercooper, 1596; Metius, Adriaan, Arithmetica et geometrice practica, Franeker, Rombertus Doyema, 1611; Snell, Willebrord, Cyclometricus, De circuli dimensione secundum 
propuestas de cuadraturas que pueden encontrarse en esos escritos son de muy distinta naturaleza. En este sentido, podría elaborarse una clasificación entre los tipos de cuadraturas propuestas en este siglo. Recientemente, Jesper Lützen ha llevado a cabo una clasificación de esta naturaleza, que es bastante compleja, estableciendo tres criterios de distinción: ${ }^{25}$ (1) el tipo de solución, esto es, si se trata de una construcción efectivamente geométrica o si, dejando de lado la cuestión de la constructibilidad geométrica de un cuadrado que sea igual al círculo o de la circunferencia extendida en línea recta, se propone una solución algebraica o aritmética, lo que es el caso de la mayoría de las propuestas del siglo XVII; (2) si el tipo de cuadratura que se ofrece nos da un resultado que es aproximado o exacto; (3) si se provee la determinación del área o circunferencia de la totalidad del círculo en términos de su radio (en este caso, tendríamos una cuadratura definida), o si se busca determinar el área o el largo del arco de cualquier sector del círculo (es decir, una cuadratura indefinida). Más aún, podrían llevarse a cabo nuevas subdivisiones teniendo en cuenta estos criterios, lo que nos muestra un panorama bastante complejo. Afortunadamente para nosotros, Leibniz hizo su propia clasificación de cuadraturas, con el objetivo fundamental de señalar lo que la suya tiene de específico. Esto lo encontramos en el Praefatio opusculi de Quadratura Circuli Arithmetica, ${ }^{26}$ texto que, en su calidad de prefacio, introduce algunas precisiones de naturaleza histórica y teórica que en buena medida proveen el marco en el que desarrolla el tratado sobre la cuadratura aritmética del círculo, de la elipse y de la hipérbola (o sea, DQA). Posiblemente, Leibniz propuso esta clasificación a partir de una observación de Oldenburg, a saber, que considere atentamente si efectivamente logró una

Logistarum abacos, \& ad Mechanicem accuratisima; atque omnium parabilissima, Leiden, ex officina Elzeviriana, 1621; Huygens, Christiaan, De circuli magnitudine inventa, 1654, en: Oeuvres complètes de Christiaan Huygens (ed. Société Hollandaise des Sciences), Amsterdam, Swets \& Zeitlinger N.V., 1910, vol. 12; Gregory, James, Vera circuli et hyperbolae quadratura, Padua, Paolo Frambotto, 1667.

${ }^{25}$ Lützen, Jesper, "17th century arguments for the impossibility of the indefinite and the definite circle quadrature", Revue d'Histoire des Mathématiques, 20, 2014, pp. 215-218. ${ }^{26}$ A VII 6, 169-177. Hay traducción al español de este texto en Leibniz, G. W., Sobre los Infinitos, prólogo, selección, traducción y notas de Oscar Esquisabel y Federico Raffo Quintana, Buenos Aires, Centro de Investigaciones Filosóficas, 2019, pp. 59-72. 
cuadratura exacta del círculo. ${ }^{27}$ Como veremos, la ordenación de las cuadraturas de Leibniz es también bastante compleja. Tengamos en cuenta el siguiente cuadro:

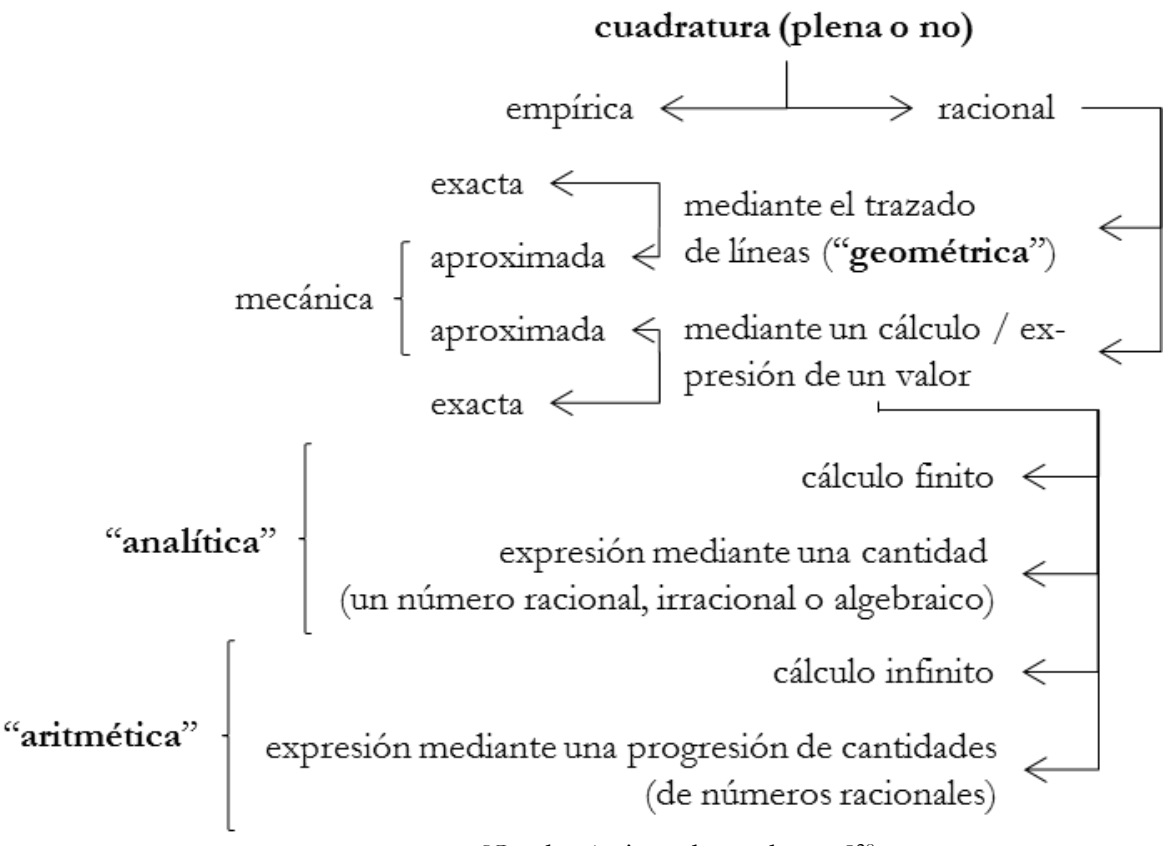

[Cuadro 1: tipos de cuadratura] ${ }^{28}$

${ }_{27}$ Crippa, Davide, Impossibility results: from geometry to analysis; a study in early modern conceptions of impossibility, Tesis doctoral, Université Paris Diderot Paris 7, 2014, pp. 414415.

28 A diferencia, Crippa incluye las cuadraturas analíticas dentro de las geométricas (Crippa, Davide, "Leibniz and the impossibility of squaring the circle", en Pisano, Raffaele, Fichant, Michel, Bussotti, Paolo y Oliveira, Agamenon R. E. (eds.), The Dialogue between Sciences, Pbilosophy and Engineering. New Historical and Epistemological Insights. Homage to Gottried W. Leibniz 1646-1716, Londres, College Publications, 2017, p. 108). No obstante, considero que esta no es la visión de Leibniz al menos por dos razones. En primer lugar, como veremos más adelante, por la descripción que hace Leibniz de una cuadratura perfecta, es decir, la que sea simultáneamente analítica y lineal, es decir, que se construye mediante líneas ecuables reductibles a 
Como vemos, ante todo hay dos grandes géneros de cuadraturas, las empíricas y las racionales. ${ }^{29}$ Una cuadratura empírica es la que se obtendría mediante un procedimiento no geométrico que nos exige llevar adelante ciertos experimentos. Por ejemplo, si pusiéramos a girar una rueda en un plano, al cabo de una vuelta completa, tendríamos, en el plano, una línea recta igual a la circunferencia de la rueda. Si tenemos en cuenta la primera proposición del Medida del círculo de Arquímedes, con la medida obtenida podríamos construir un triángulo que sea igual al círculo (es decir aquel cuya base sea la circunferencia extendida en línea recta y cuya altura sea el radio del círculo). Ahora bien, este tipo de procedimientos carece de interés para los geómetras, porque buscan una línea recta que sea igual a la circunferencia o el lado de un cuadrado que sea igual a un círculo mediante procedimientos que no sean empíricos, o mejor dicho, "gracias a cierta técnica, regla o instrumento, que tenga la capacidad de rectificar". ${ }^{30}$ Esto nos deja solamente con las cuadraturas 'racionales'. Dentro de las cuadraturas racionales, hay que hacer algunas precisiones. Por lo pronto, hay dos subdivisiones que se solapan: las cuadraturas racionales pueden ser (o aspirar a ser) exactas o aproximadas, y en ambos casos puede buscarse la cuadratura mediante 'el trazado de líneas' o mediante 'un cálculo o expresión de un valor'. A las cuadraturas que se buscan mediante el trazado de líneas, esto es, con regla y compás, Leibniz las llama geométricas. ${ }^{31}$ Por otra parte, la postulación de que podemos buscar una cuadratura mediante un cálculo, y no solamente mediante procedimientos puramente geométricos, acentúa una característica distintiva del siglo XVII en relación con el problema de la cuadratura del círculo, tal como mencionamos antes. En las cuadraturas que se buscan mediante un cálculo o expresión de un valor, hay una doble distinción a considerar: en primer lugar, el cálculo que se emplee puede ser finito o infinito; en segundo lugar, puede expresarse mediante

ecuaciones de dimensiones conocidas (A VII 6, 175). Esto parece implicar que no son lo mismo. En segundo lugar, porque para Leibniz no todo lo analítico es geométrico, ya que hay algunas cantidades que no pueden exhibirse dibujando líneas.

${ }^{29}$ Omito aquí la distinción entre cuadratura plena (cuadratura del círculo y de todas sus partes, es decir, segmentos y sectores) o menos que plena.

30 A VII 6, 170. “(...) certa arte ac regula, instrumentove quod dirigere sit in potestate (...)”. Traducción: Leibniz, G. W., Sobre los Infinitos, p. 61.

${ }^{31}$ A VII 6, 175. 
una cantidad o mediante una progresión de cantidades. Cuando se busca expresar el valor para el área del círculo mediante un número, sea racional, irracional o algebraico, tenemos una cuadratura analítica. Leibniz señala, en rigor, que la expresión es analítica. Ahora bien, si el valor se expresa no mediante un número, sino mediante una progresión, y más aún, cuando se expresa el valor mediante una progresión de números racionales, lo que tenemos es una cuadratura aritmética.

Antes de proseguir examinando la distinción entre la expresión de un valor mediante una cantidad o mediante una progresión de cantidades, consideremos la distinción entre cuadraturas exactas y aproximadas. Leibniz llama a las cuadraturas aproximadas mecánicas, debido a que nos ofrecen un procedimiento para aproximarnos sucesivamente a la cuadratura. Leibniz señala ejemplos de cuadraturas aproximadas tanto geométricas como de las que emplean un cálculo, como las cuadraturas de Arquímedes, A. Metius, L. van Ceulen, J. Gregory y otros. ${ }^{32}$ Varias de estas cuadraturas aproximadas han dado por resultado proporciones que nos permiten calcular con un grado de precisión relativo, entre las que Leibniz destaca las proporciones de los primeros tres autores recién mencionados. La proporción que Arquímedes presentó en la segunda proposición de Medida del círculo implica que la circunferencia de un círculo es a su diámetro como 22 a 7. Esto nos muestra un valor para la cuadratura realmente cercano a $\pi$, aunque no lo expresa de manera exacta. Por eso, según Leibniz, esta proporción es útil para cuestiones de poca importancia, es decir, aquellas en las que no se requiere una precisión extrema. La proporción de Metius es que la circunferencia es al diámetro como 355 a 113. Esta proporción es realmente más precisa que la de Arquímedes, aunque tampoco es exacta. Por eso mismo, para Leibniz esta proporción es útil para cuestiones 'de mediana importancia', es decir, para aquellas que requieren un poco más de precisión que las anteriores. Finalmente, Leibniz menciona que la proporción dada por Ludolph van Ceulen es útil para tratar cuestiones que

${ }^{32}$ Leibniz se refiere, en especial, a Medida del círculo de Arquímedes (Arquímedes, Tratados I. Sobre la esfera y el cilindro. Medida del Circulo. Sobre los conoides y esferoides, introducción, traducción y notas de Paloma Ortíz García, Madrid, Gredos, 2005, pp. 243-249), a Arithmetica et geometria practica de Adriaan Metius (1611), a Vanden Circkel de Ludolph van Ceulen (1596) y a Vera circuli et hyperbolae quadratura de James Gregory (1667). 
son muy importantes. Dicha proporción (a la que Leibniz se refiere indirectamente en este texto, de manera que no la menciona explícitamente) implica que el círculo es al diámetro un poco más que 314.159.265.358.979.323.846 a 100.000.000.000.000.000.000.33

Todas estas cuadraturas aproximadas nos ofrecen resultados con diversos grados de precisión, aunque ninguna de ellas adquiere un grado de rigurosidad máximo. Naturalmente, el resultado buscado en última instancia para el problema de la cuadratura es exacto. La cuadratura exacta es, para Leibniz, aquella en la que se exhibe la magnitud buscada del círculo no ya de manera aproximada, sino con total precisión, sea que esto se obtenga mediante 'el trazado de líneas' o mediante 'la expresión de un valor'. En este sentido, a priori no se excluye la posibilidad de que haya una cuadratura exacta mediante procedimientos puramente geométricos. Ahora bien, en el caso de las cuadraturas buscadas mediante la expresión de un valor, Leibniz señala que el valor buscado podría expresarse o bien mediante una cantidad, o bien mediante una progresión de cantidades (aunque, como veremos, cumpliendo ciertas condiciones). Como señalamos antes, la posibilidad de ser expresado mediante una cantidad involucra la idea de poder dar una cuadratura mediante un cálculo finito, de manera que la cantidad quede expresada mediante un número racional, irracional o algebraico, dado por una ecuación.

Por su parte, Leibniz describe las cuadraturas exactas que expresan el valor mediante una progresión de cantidades de este modo: "cuando alguien muestra que una progresión, de la cual se da una regla para continuarla al infinito, tomada toda a la vez, expresa exactamente el valor del arco o del círculo". ${ }^{34}$ Como vemos, hay dos condiciones que requiere una cuadratura aritmética para ser exacta. ${ }^{35}$ En primer lugar, se requiere disponer de la regla para continuar la progresión al infinito. Es decir, se requiere que sea una serie regular. Debemos conocer la ley de la serie, o, como Leibniz suele llamarlo en

33 Así es presentado por Metius al final de la octava sección del capítulo VII de la primera parte de Geometriae practica, 1611, p. 45.

${ }^{34}$ A VII 6, 174. "Per progressionem, si quis ostendat progressionem quandam, cujus continuandae in infinitum regula datur, totam simul sumtam arcus vel circuli valorem exacte exprimere". Traducción: Leibniz, G. W., Sobre los Infinitos, p. 67.

${ }^{35} \mathrm{He}$ abordado con más detalle las condiciones de completitud y regularidad en Raffo Quintana, Federico, "Leibniz on the requisites of an exact arithmetical quadrature", Studies in History and Philosophy of Science, 67, 2018, pp. 65-73. 
textos de este período, el fundamentum progressionis. ${ }^{36}$ Esto nos permitiría visualizar el modo como procede la sucesión. La regularidad es en lo que falla, por ejemplo, la proporción propuesta por Ludolph van Ceulen, pues no hay una regla de este tipo. Idealmente, para Leibniz la regla tiene que ser lo más simple posible, y en este sentido incluso señala que, si hubiera una regla para la proporción de Ludolph van Ceulen, posiblemente no sea muy simple (o por lo menos, como veremos, tan simple como la que él propone). En segundo lugar, la serie cuya regla conocemos, debe ser tomada toda a la vez, de modo que hay una suposición de completitud de la serie. En otras palabras, una serie puede expresar un valor de manera exacta solamente si se asume que están dados todos los términos. Esto no significa que para Leibniz una serie tenga un número infinito de términos, pues un número infinito es contradictorio. No obstante, en las sumas de series decrecientes, podemos proceder como si hubiera un último término (lo que no hay, y por ello tal término infinitamente pequeño es una ficción), sobre la base de que entendamos que, para cualquier término, no importa cuán pequeño sea, podemos encontrar siempre otro menor. ${ }^{37} \mathrm{Si}$ no se presupusiera la completitud, el valor no sería exacto, sino aproximado. Así, sobre la base de estas condiciones, en el caso de que la progresión que tengamos sea de números racionales, tendríamos una cuadratura aritmética exacta como la que Leibniz propone:

como cuando digo: si el cuadrado del diámetro es 1 , el círculo se iguala a la totalidad de la progresión de las fracciones que tienen a la unidad como numerador y a los impares como denominador alternativamente afirmadas y negadas, a saber, $\frac{1}{1}-\frac{1}{3}+\frac{1}{5}-\frac{1}{7}+\frac{1}{9}-\frac{1}{11}$ etc. al infinito. ${ }^{38}$

\footnotetext{
36 Por ejemplo, A VII 3, 35 y A VII 6, 600.

37 Sobre esta cuestión, véase Arthur, Richard T. W., "Leibniz's syncategorematic infinitesimals", Archive for History of Exact Sciences, 67, 2013, pp. 553-593. Recientemente, Arthur ha ofrecido una visión aún más amplia y completa de la concepción 'sincategoremática' del infinito en Leibniz en Arthur, Richard T. W., "Leibniz's Syncategorematic Actual Infinite", en Nachtomy, Ohad y Winegar, Reed (eds.), Infinity in Early Modern Philosophy, Springer, 2018, pp. 155-179.

38 A VII 6, 174. “(...) ut cum dico[:] Si quadratum diametri sit 1, Circulum aequari toti progressioni fractionum sub unitate imparium alternis affirmatarum et negatarum,
} 
Como vemos, Leibniz presentó una cuadratura del círculo mediante números racionales, pues la serie se compone de números fraccionarios. No obstante eso, reconoce que sería bellísimo encontrar la cuadratura mediante números enteros, lo que podría lograrse si alguien encontrara una progresión de caracteres que nos permita continuar al infinito la proporción de Ludolph van Ceulen. A pesar de ello, Leibniz es consciente de que esta regla (supuesto que sea posible obtenerla) sería dificilísima, por lo cual la 'belleza' de esta regla implicaría una falta de simplicidad. En este sentido, parece que el criterio de simplicidad prima por sobre el de belleza. En este caso, la simplicidad se observa en la regularidad de la serie.

En síntesis, más allá de las primeras clasificaciones, Leibniz distingue tres cuadraturas de interés para los geómetras: las geométricas (que proceden mediante el trazado de líneas), las analíticas (numéricas que buscan expresar el valor mediante un cálculo finito, sea de números racionales, irracionales o algebraicos) y las aritméticas (numéricas que proceden mediante un cálculo infinito de números racionales). De este último tipo es la cuadratura que Leibniz presentó. En este sentido, cree haber ofrecido, por primera vez, una cuadratura que no es aproximada, sino exacta. Así, si buscáramos en el texto de Leibniz ejemplos de cuadraturas exactas, solamente encontraríamos su propuesta. Ahora bien, esto no significa que la cuadratura aritmética sea perfecta. Una cuadratura perfecta sería, para Leibniz, aquella que sea, al mismo tiempo, geométrica y analítica. Así, sería perfecta la cuadratura que pueda construirse mediante líneas ecuables, trazadas mediante regla y compás, y que puedan expresarse dichas líneas mediante ecuaciones de grado finito. No obstante, Leibniz considera, retomando la propuesta de James Gregory, que una cuadratura de este tipo es imposible (aunque, para Leibniz y otros autores, la demostración de Gregory en este asunto no es absolutamente conclusiva). ${ }^{39}$ Esto explica por qué para Leibniz su cuadratura aritmética, aunque no es perfecta (porque no es ni analítica ni geométrica), es la mejor que podemos tener (como busca mostrar en la proposición LI de $D Q A$ ).

\section{La imposibilidad de infinitizar el producto infinito de Wallis}

nempe $\frac{1}{1}-\frac{1}{3}+\frac{1}{5}-\frac{1}{7}+\frac{1}{9}-\frac{1}{11}$ etc. in infinitum (...)". Traducción: Leibniz, G. W., Sobre los Infinitos, p. 67.

${ }^{39} \mathrm{Al}$ respecto, véase Crippa, Impossibility results, pp. 342-354 y 422-428. 
La clasificación propuesta por Leibniz que recién describimos tiene una curiosidad significativa: Leibniz no menciona a Wallis ni una sola vez en el todo el Praefatio. Esto es extraño por varias razones: primero, porque en 1676 Leibniz ya conocía varios trabajos de Wallis, tal como mencionamos al comienzo; segundo, porque el producto infinito, si tenemos en cuenta la clasificación de Leibniz, parece darnos una cuadratura racional mediante un cálculo infinito de números racionales. Más aún, como señalamos antes, si el producto infinito de Wallis es $\frac{3 \times 3 \times 5 \times 5 \times 7 \times 7 \text { etc. }}{2 \times 4 \times 4 \times 6 \times 6 \times 8 \text { etc. }}=\frac{4}{\pi}$ y la serie infinita de Leibniz es $\frac{1}{1}-\frac{1}{3}+\frac{1}{5}-\frac{1}{7}+$ $\frac{1}{9}-\frac{1}{11}=\frac{\pi}{4}$, entonces tendríamos un resultado equivalente. En otras palabras, si esto es así, podría pensarse que Wallis también proveyó una cuadratura aritmética exacta, por lo que tendría incluso lugar la siguiente pregunta: el producto infinito de Wallis, ¿no es más simple que la serie de Leibniz? Esto es relevante, pues, supuesto que lo fuera, y según los criterios del mismísimo Leibniz, su cuadratura no sería la mejor que podamos obtener.

Ahora bien, Leibniz tiene razones para evitar argumentos de esta naturaleza. Por lo pronto, considera que, hasta ese momento, no se había conseguido nada considerable en la búsqueda de una cuadratura exacta. Más aún, lo único que Leibniz dice que es digno de ser destacado es la 'bella aproximación' de Wallis mediante números racionales. ${ }^{40}$ En este sentido, cuando, en el Preafatio, Leibniz señala, como ejemplos de cuadraturas aproximadas, los trabajos de Arquímedes, Metius, van Ceulen, Gregory "y otros", podríamos incluir el producto de Wallis. Más aún, en la Arithmetica infinitorum, Wallis mismo señala que el producto tiene un carácter aproximado, pues nos permite expresar la cuadratura del círculo "en cuanto pueda realizarse por la naturaleza de los números". ${ }^{41}$ En la literatura secundaria, es usual señalar que buena parte de las críticas que recibió Wallis en su siglo está vinculada con el uso que hizo de la inducción en matemática infinita. ${ }^{42}$ Esto es efectivamente

\footnotetext{
40 A VII 6, 89.

${ }^{41}$ Wallis, Arithmetica infinitorum, p. 177. “(...) quatenus ipsa numerorum natura ferat”.

42 Por ejemplo: Jesseph, Douglas, Squaring the Circle. The War between Hobbes and Wallis, Chicago \& Londres, The University of Chicago Press, 1999, pp. 177-178; Beeley, Philip, "Nova methodus investigandi'. On the Concept of Analysis in John Wallis's
} 
cierto en el caso de Leibniz, como puede observarse, por ejemplo, cuando señala que "Wallis procede por no sé qué método de inducción en su Arithmetica infinitorum". ${ }^{43}$ No obstante, Leibniz tiene también razones para sostener que, si se infinitizara el producto - de tal manera que proveyera exactamente el número $\square-$, el resultado que se seguiría sería absurdo. Esto justificaría, entonces, no sólo que para Leibniz el producto de Wallis no ofrece un resultado exacto, sino también que no sería posible que exhiba con exactitud.

A finales del verano de 1676, Leibniz redacta un breve apunte en el que examina con cierto detenimiento el producto de Wallis. ${ }^{44}$ Leibniz considera, en especial, qué implica que el producto infinito se iguale al número cuadrado, esto es, que $\frac{1 \times 3 \times 3 \times 5 \times 5 \times 7 \times 7 \times 9 \times 9 \times 11 \times 11 \text { etc. }}{2 \times 4 \times 4 \times 6 \times 6 \times 8 \times 8 \times 10 \times 10 \times 12 \times 12 \text { etc. }}=\square .45$ En particular, su atención se centra en el etc. que marca la continuación infinita de la fracción, sin la cual la igualdad no se daría. Esta manera de abordar el producto, es decir, como igualable al número $\square$ si se lo extendiera al infinito, no es una arbitrariedad de Leibniz, pues Wallis sugiere esto cuando señala que: "decimos que esta fracción, $\frac{3 \times 3 \times 5 \times 5 \times 7 \times 7 \text { etc. }}{2 \times 4 \times 4 \times 6 \times 6 \times 8 \text { etc. }}$, continuada al infinito, es precisamente el mismísimo número buscado $\square$, respecto del cual 1 se comporta del mismo modo que el Círculo al cuadrado del diámetro". ${ }^{46}$ Como vimos en la sección anterior, Leibniz entiende que uno de los requisitos que debe darse para que haya una verdadera y exacta igualdad es la suposición de completitud de la serie infinita (en este caso, del producto). De esta manera, en síntesis, el examen que

Mathematical Writings", Studia Leibnitiana, 45/1, 2013, pp. 54-55; Crippa, Impossibility results, p. 416.

${ }^{43}$ A VII 3, 183. "Wallis procedit nescio qua methodo inductionis, in sua Arithmetica infinitorum".

${ }^{44}$ A VII 3, 824-826.

${ }^{45}$ Notemos que hay diferencias entre el modo como Wallis y Leibniz presentan la fracción. Por ejemplo, en el caso de Leibniz, en el numerador, la unidad es el primer término y, en el denominador, los últimos dos términos son iguales. Estas cosas, como vimos, no se siguen de la regla de Wallis para generar el producto. Como veremos, estas diferencias fueron significativas para el planteo de Leibniz.

${ }^{46}$ Wallis, Arithmetica infinitorum., p. 180. "Dicimus, fractionem illam $\frac{3 \times 3 \times 5 \times 5 \times 7 \times 7 \text { etc. }}{2 \times 4 \times 4 \times 6 \times 6 \times 8 \text { etc. }}$. infinitum continuatam, esse ipsissimum quasitum numerum $\square$ praecise; ad quem ita se habet 1, ut Circulus ad Quadratum diametri”. 
Leibniz hizo del producto infinito de Wallis consistió en ver qué se sigue de suponer que el producto es infinito, es decir, que está dado de manera completa. Mediante una serie de operaciones algebraicas, Leibniz concluye que la infinitización del producto de Wallis nos lleva a la conclusión absurda de que $\sqrt{2 \square}$ sería una cantidad infinitamente pequeña. El hilo de su razonamiento es el siguiente:

1.

Si $\frac{1 \times 3 \times 3 \times 5 \times 5 \times 7 \times 7 \times 9 \times 9 \times 11 \times 11 \text { etc. }}{2 \times 4 \times 4 \times 6 \times 6 \times 8 \times 8 \times 10 \times 10 \times 12 \times 12 \text { etc. }}=\square$, entonces la primera fracción, es decir $\frac{1}{2}$, se traspasa al otro lado de la ecuación de la manera correspondiente (es decir, el denominador multiplicando y el numerador dividiendo). De allí se obtiene que $\frac{3 \times 3 \times 5 \times 5 \times 7 \times 7 \times 9 \times 9 \times 11 \times 11 \text { etc. }}{4 \times 4 \times 6 \times 6 \times 8 \times 8 \times 10 \times 10 \times 12 \times 12 \text { etc. }}=$ $2 \square$.

2. Leibniz parece considerar, tanto en los numeradores como en los denominadores, los pares de números iguales que se multiplican como cuadrados, es decir, $\frac{3 \times 3 \times 5 \times 5 \times 7 \times 7 \times 9 \times 9 \times 11 \times 11 \text { etc. }}{4 \times 4 \times 6 \times 6 \times 8 \times 8 \times 10 \times 10 \times 12 \times 12 \text { etc. }}=$

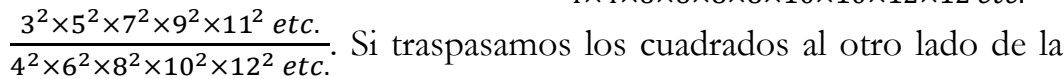
ecuación, obtendremos que $\frac{3 \times 5 \times 7 \times 9 \times 11 \text { etc. }}{4 \times 6 \times 8 \times 10 \times 12 \text { etc. }}=\sqrt{2 \square}$.

3. Leibniz simplifica el producto en el sentido de que, por ejemplo, $\frac{3}{6}=\frac{1}{2}$, o $\frac{5}{10}=\frac{1}{2}$, y así sucesivamente. De allí resulta que $\frac{1 \times 1 \times 1 \text { etc. }}{4 \times 2 \times 8 \times 2 \times 12 \times 2 \text { etc. }}=\sqrt{2 \square}$, y, por lo tanto, $\frac{1}{8 \times 16 \times 24 \text { etc. }}=\sqrt{2 \square}$.

4. Ahora bien, como denominador puede hacerse infinitamente grande, $\sqrt{2 \square}$ es una cantidad infinitamente pequeña, lo que es un absurdo. En palabras -un poco crípticas- de Leibniz:

Pero de aquí concluyo que la expresión de Wallis sin dudas puede tenerse por una aproximación de la cual la continuabilidad al infinito es evidente, pero no por una expresión exacta mediante una serie infinita considerada completamente de una vez, pues, expresando mediante la supuesta continuación al infinito, surge un absurdo, pues pueden eliminarse todos los números del numerador, puesto que tenemos el múltiplo de ellos en el denominador, si los entendiéramos a ambos [es decir, numerador y denominador] continuados al infinito, y resultaría así 
que $\sqrt{2 \square}$ es igual a $\frac{1}{4 \times 2 \times 8 \times 2 \times 12 \times 2 \text { etc. }}$ o bien a $\frac{1}{8 \times 16 \times 24 \text { etc. }}$, lo que es absurdo; en efecto, $\sqrt{2 \square}$ se haría una cantidad infinitamente pequeña. Del mismo modo, sucedería que $2 \square$ es igual a $\frac{1}{64 \times 256 \times 576 \text { etc. }}$, es decir, $2 \square$ es igual a $\frac{1}{64 \times 164 \times 464 \times 9 \text { etc. }}{ }^{47}$

Como vemos, hay una distinción entre interpretar que el producto es infinito e interpretar que es continuable al infinito. En el primer caso, estaríamos tomando el producto como una totalidad, es decir, 'completamente de una vez'; en el segundo, estaríamos señalando la posibilidad de extender continuamente el producto de manera indefinida. De esta manera, se entiende por qué Leibniz no contempla que el producto de Wallis provea un resultado exacto, pues si lo tomáramos en su totalidad (es decir, si dijéramos que es infinito), se seguiría un absurdo (que un poco más adelante examinaremos con más detalle). De allí que Leibniz extraiga dos conclusiones sobre el producto de Wallis:

1. Debe decirse más bien que el producto de Wallis se aproxima continuamente al número $\square$ por medio de estos valores: $\frac{3 \times 3}{2 \times 4} ; \frac{3 \times 3 \times 5 \times 5}{2 \times 4 \times 4 \times 6}$; $\frac{3 \times 3 \times 5 \times 5 \times 7 \times 7}{2 \times 4 \times 4 \times 6 \times 6 \times 8} ;$ y así sucesivamente, con tal que entendamos que cada una de estas instancias posee un número finito de términos.

2. En consecuencia, en sentido estricto, el producto no es infinito: "Y por lo tanto, la serie de Wallis debe ser llamada propiamente finita indefinida, es decir, que debe concebirse que tiene un fin en alguna parte, aunque no se exprese dónde lo tiene". 48

${ }^{47}$ A VII 3, 824. "Sed hinc colligo expressionem Wallisianam posse quidem haberi pro appropinquatione, cuius apparet continuabilitas in infinitum, sed non pro exacta expressione per infinitam seriem consideratam semel in universum, nam exprimendo per suppositam continuationem in infinitum, oritur absurdum, nam omnes numeri numeratoris tolli possunt, quia habentur eorum multipli in nominatore, si uterque in infinitum continuatus intelligitur, et ita staret $\sqrt{2 \square}$ aeq. $\frac{1}{4 \times 2 \times 8 \times 2 \times 12 \times 2 \text { etc. }}$ vel $\frac{1}{8 \times 16 \times 24 \text { etc. }}$ quod est absurdum, fieret enim $\sqrt{2 \square}$ quantitas infinite parva. Similiter fieret $2 \square$ aeq. $\frac{1}{64 \times 256 \times 576 \text { etc. }}$ seu $2 \square$ aeq. $\frac{1}{64 \times 164 \times 464 \times 9 \text { etc. }}$.

48 A VII 3, 825; la cursiva es mía. "Et proinde series Wallisiana est proprie appellanda finita indefinita, seu quae alicubi finienda concipi debet, licet non exprimatur ubi". 
Ahora bien, el planteo de Leibniz deja sin especificar, al menos en este lugar, un aspecto muy relevante de su planteo, a saber, por qué razón es un absurdo que $\sqrt{2 \square}$ sea una cantidad infinitamente pequeña. En otras palabras, la lectura de Leibniz presupone que las cantidades infinitamente pequeñas son absurdas.

\section{La naturaleza de las cantidades infinitamente pequeñas}

La cuestión de las cantidades infinitamente pequeñas es uno de los aspectos más destacados de la matemática infinita leibniziana de los últimos años del período parisino. Como muestra la literatura sobre esta cuestión, ${ }^{49}$ Leibniz sostuvo que las cantidades infinitamente pequeñas, así como también las cantidades infinitas, son ficticias. Leibniz le reconoce a estas cantidades una inmensa utilidad, entre otras cosas, tanto para el descubrimiento de nuevas verdades como para su demostración. ${ }^{50}$ En el primer caso, Leibniz señala que el campo de la demostración se amplía enormemente por el mero hecho de sostener que toda figura curvilínea es un polígono con un número infinito de lados, de magnitud infinitamente pequeña. ${ }^{51}$ No es mi intención aquí detenerme a examinar de qué manera impacta el empleo de cantidades ficticias en el descubrimiento. Mi intención es más bien explicar por qué las cantidades infinitamente pequeñas son absurdas, en vistas a entender el argumento leibniziano sobre el producto de Wallis que recién expuse.

Ahora bien, el hecho de que los geómetras entiendan el provecho de introducir cantidades ficticias tan pronto como conciban las figuras curvilíneas como polígonos infinitángulos, parece proveernos, al mismo tiempo, de una rápida descripción de la naturaleza de las cantidades ficticias. Como explica en

49 Entre muchos otros: Arthur, R., “Leibniz's syncategorematic infinitesimals”, pp. 553-593; Katz, Mikhail y Sherry, David, “Leibniz’s infinitesimals: their fictionality, their modern implementations, and their foes from Berkeley to Russell and beyond", Erkenntnis, 78/3, 2013, pp. 571-625; y Knobloch, Eberhard, "Leibniz and the infinite", Quaderns d'Història de l'Enginyeria, XVI, 2018, pp. 11-31. Ahora bien, no hay unanimidad sobre el modo de comprender la naturaleza y el uso de cantidades ficticias en Leibniz, por lo que en los textos que aquí señalamos se pueden obtener distintas lecturas.

${ }^{50}$ A VII 6, 585.

51 A VII 6, 586. 
el escrito Numeri infiniti de abril de 1676, desde un punto de vista cognitivo, el polígono infinitángulo es el que la mente se imagina que es el último de la serie de los polígonos, como si fuera de hecho posible que haya una última figura poligonal. ${ }^{52}$ En consecuencia, en dicho polígono, si fuera posible (lo que no implica que sea posible), se daría el mayor número de lados (un número infinito, es decir, máximo), del mismo modo que los lados de dicho polígono serían los menores. Si un polígono infinitángulo fuera posible, habría un número máximo y una línea mínima. No obstante, para Leibniz "No hay un número máximo y no hay una línea mínima". ${ }^{53}$ En este sentido, es metafísicamente imposible. Esto explica por qué para Leibniz son imposibles no sólo los problemas que arriban a una ecuación contradictoria, sino también aquellos que se resuelven "en una cantidad imaginaria, de la cual no puede entenderse posición alguna". ${ }^{54}$

Este doble aspecto del polígono infinitángulo -su utilidad para la geometría y su imposibilidad metafísica- queda remarcado en un pasaje del diálogo Pacidius Philalethi (que Leibniz finalmente descartó y reemplazó por otro): "Del mismo modo, el polígono regular de infinitos lados no puede tenerse metafísicamente por un círculo, aunque en Geometría se tenga por un círculo, si el error es menor que cualquier número que podamos expresar". ${ }^{55}$ Más aún, en el mismo escrito, Leibniz indica la razón de la imposibilidad de las cantidades infinitamente pequeñas ficticias:

Yo sin duda admitiría estos espacios y tiempos infinitamente pequeños en Geometría, a los fines de la invención, aunque fueran imaginarios. Pero me pregunto si acaso pueden ser admitidos en la naturaleza. En efecto, de allí parecen originarse líneas rectas infinitas terminadas por ambos lados, como mostraré en otra parte, lo cual es absurdo. Además, ya que pueden asumirse al infinito otras cosas infinitamente pequeñas

52 A VI 3, 498. Hay traducción de este texto en Leibniz, G. W., Sobre los infinitos, pp.4158.

53 A VI 3, 502. Traducción: Leibniz, G. W., Sobre los infinitos, p. 54.

${ }^{54}$ A VI 3, 464. (...) "cum resolvitur in imaginariam quantitatem, cuius nullus intelligi potest situs".

55 A VI 3, 569. "Quemadmodum polygonum regulare infinitorum laterum pro circulo metaphysice haberi non potest, tametsi in Geometria pro circulo habeatur, ob errorem minorem quam ut numero ullo a nobis exprimi possit". 
aún menores que otras, nuevamente no puede ofrecerse una razón de por qué se asumen unas más que otras; pues nada sucede sin razón. ${ }^{56}$

De esta manera, en pocas palabras, una cantidad infinitamente pequeña no es posible, pues es siempre posible pensar que hay una cosa menor que otra al infinito. En consecuencia, Leibniz parece estar diciendo que para toda cantidad posible vale lo que posteriormente se conoció como el axioma de Arquímedes, o mejor, la formulación equivalente según la que, supuestas dos magnitudes que no sean iguales, si a la mayor de ellas se le quitara una parte que sea mayor que su mitad, nuevamente a la parte restante se le quitara una parte que sea mayor que su mitad, y se replicara sucesivamente el mismo procedimiento, entonces se obtendría finalmente una magnitud menor que la magnitud más pequeña de las dos consideradas inicialmente. ${ }^{57}$ Por contraposición, sería metafísicamente imposible una cantidad en la que no se cumpla el axioma. Así, una cantidad infinitamente pequeña terminada, como el lado del polígono infinitángulo, sería una cantidad para la cual no puede hallarse otra menor. Como vimos, para Leibniz esta cantidad no es posible.

Con esto en mente, la conclusión de Leibniz sobre el producto infinito de Wallis es clara. Si tenemos que $\sqrt{2 \square}=\frac{1}{8 \times 16 \times 24 \text { etc. }}$ y el denominador es infinitamente grande, $\sqrt{2 \square}$ deviene una cantidad infinitamente pequeña. En este caso, no introducimos una ficción que nos permita descubrir o demostrar, sino que, más bien, concluimos que hay una cantidad infinitamente pequeña que

${ }^{56}$ A VI 3, 564-565. "Ego spatia haec et tempora infinite parva in Geometria quidem admitterem, inventionis causa, licet essent imaginaria. Sed an possint admitti in natura delibero. (...) Praeterea cum infinitae parvae quoque aliae aliis minores assumi possint in infinitum, rursus non potest ratio reddi, cur aliae prae aliis assumantur; nihil autem fit sine ratione".

${ }^{57}$ Esta formulación puede hallarse en los Elementos, X, 1 (Euclides, Elementos. Libros XXIII (traducción y notas de María Luisa Puertas Castaños), Madrid, Gredos, 1996). Otra formulación conocida es que, dadas dos cantidades $x$ e $y$ tales que $x<y$, es posible hallar un número natural $n$ de modo que $n x>y$. Véase Levey, Samuel, "Archimedes, Infinitesimals and the Law of Continuity: On Leibniz's Fictionalism”, en Goldenbaum, Ursula y Jesseph, Douglas (eds.), Infinitesimal Differences: Controversies between Leibniz and his Contemporaries, Berlin \& New York, Walter de Gruyter, 2008, p. 113; y Arthur, R., "Leibniz’s syncategorematic infinitesimals", pp. 559-561. 
es equivalente a la raíz cuadrada del doble del número cuadrado. Ahora bien, si estas cantidades son imposibles, la conclusión que se deduce, según Leibniz, de infinitizar el producto de Wallis, no puede defenderse.

\section{Conclusiones}

Si tenemos en cuenta el argumento de Leibniz por el que el producto de Wallis no puede infinitizarse y la justificación que dio de ello, podemos entender un poco mejor algunas de las razones por las que excluyó el planteo del matemático británico cuando propuso la tabla de cuadraturas. Primero, queda claro por qué para Leibniz la de Wallis es una cuadratura aproximada: para que una cuadratura aritmética, por medio de series infinitas de números racionales, provea un resultado exacto, debe suponerse la completitud del proceso infinito, lo que para Leibniz no puede llevarse a cabo en el caso del producto de Wallis. Segundo, eso explica también por qué el hecho de que el producto de Wallis exhiba una regla para su generación parece ser irrelevante a los ojos de Leibniz: si lo mejor que puede proveer es una 'bella aproximación', no importa la sencillez de su regla (supuesto que de hecho sea sencilla), porque lo que se busca es expresar exactamente el valor para el círculo.

Ahora bien, si la lectura que proponemos es correcta, podemos extraer una aclaración sobre el empleo que Leibniz hizo de cantidades ficticias en matemática a partir de su examen del producto de Wallis. Podemos introducir, al modo de una suposición, cantidades ficticias; no obstante, no es lícito arribar, a modo de conclusión, es decir, deductivamente, a una cantidad ficticia. Esto sería equivalente a decir que arribamos a una imposibilidad. Como Leibniz suele mencionar, el empleo de cantidades ficticias sirve para abreviar las demostraciones (así como también abrevian, por cierto, el proceso de pensar, de hablar y de descubrir). ${ }^{58}$ Precisamente por eso, es posible reconstruir las demostraciones sobre cuadraturas siguiendo otros métodos, como, por ejemplo, el método de doble reducción al absurdo de Arquímedes, a saber, puesto que el resultado al que llegamos con ambos métodos es el mismo. ${ }^{59}$ Obtener como resultado de una serie de operaciones aritméticas una cantidad ficticia, sea por el método de Leibniz o por cualquier otro, sería un absurdo.

\footnotetext{
58 A VII 6, 585-586.

${ }^{59}$ A VII 6, 537.
} 
Simplificar el proceso de demostración, por otro lado, sería, en muchos casos, enormemente útil.

\section{Bibliografía}

Andersen, Kirsti, "Cavalieri's Method of Indivisibles", Archive for History of Exact Sciences, 31/4, 1985, pp. 291-367.

Andersen, Kirsti, "The Method of the Indivisibles: Changing Understandings", Studia Leibnitiana, Sonderbeft 14, 1986, pp. 14-25.

Andersen, Kirsti, “Cavalieri's Indivisibles”, en Jullien, Vincent (ed.), SeventeenthCentury Indivisibles Revisited, Dordrecht, Birkhäuser, 2015, pp. 31-55.

Arquímedes, Tratados I. Sobre la esfera y el cilindro. Medida del Círculo. Sobre los conoides y esferoides (introducción, traducción y notas de Paloma Ortíz García), Madrid, Gredos, 2005.

Arthur, Richard T. W., "Leibniz's syncategorematic infinitesimals", Archive for History of Exact Sciences, 67, 2013, pp. 553-593.

Arthur, Richard T. W., "Leibniz's Syncategorematic Actual Infinite", en Nachtomy, Ohad y Winegar, Reed (eds.), Infinity in Early Modern Philosophy, Springer, 2018, pp. 155-179.

Beeley, Philip, "Nova methodus investigandi'. On the Concept of Analysis in John Wallis's Mathematical Writings", Studia Leibnitiana, 45/1, 2013, pp. 42-58.

Cavalieri, Bonaventura, Geometria indivisibilibus continuorum quadam nova ratione promota, Boloña, 1653.

Crippa, Davide, Impossibility results: from geometry to analysis; a study in early modern conceptions of impossibility, tesis doctoral, Paris, Université Paris Diderot Paris 7, 2014.

Crippa, Davide, "Leibniz and the impossibility of squaring the circle", en Pisano, Raffaele, Fichant, Michel, Bussotti, Paolo y Oliveira, Agamenon R. E. (eds.), The Dialogue between Sciences, Philosophy and Engineering. New Historical and Epistemological Insights. Homage to Gottfried W. Leibniz. 1646-1716. Londres, College Publications, 2017. 
Euclides, Elementos. Libros X-XIII, traducción y notas de María Luisa Puertas Castaños, Madrid, Gredos, 1996.

Gregory, James, Vera circuli et hyperbolae quadratura, Padua, Paolo Frambotto, 1667.

Huygens, Christiaan, Oeuvres complètes de Christiaan Huygens (ed. Société Hollandaise des Sciences). Amsterdam, Swets \& Zeitlinger, 1910, vol. 12.

Jesseph, Douglas, Squaring the Circle. The War between Hobbes and Wallis, Chicago \& Londres, The University of Chicago Press, 1999.

Katz, Mikhail y Sherry, David, "Leibniz's infinitesimals: their fictionality, their modern implementations, and their foes from Berkeley to Russell and beyond", Erkenntnis, 78/3, 2013, pp. 571-625.

Knobloch, Eberhard, "Leibniz and the infinite", Quaderns d'Història de l'Enginyeria, XVI, 2018, pp. 11-31.

Leibniz, G. W., Sämtliche Schriften und Briefe (edición de la Academia de Ciencias de Berlin), Berlín (antes: Darmstadt; Leipzig), Walter de Gruyter Verlag (antes: Otto Reichl Verlag; Akademie-Verlag), 1923 y ss. (Citado como A, seguido de la serie (en números romanos), del volumen (en números arábigos) y del número de página. Por ejemplo: A VII 6, 600)

Leibniz, G. W., Sobre los Infinitos (prólogo, selección, traducción y notas de Oscar Esquisabel y Federico Raffo Quintana). Buenos Aires, Centro de Investigaciones Filosóficas, 2019.

Levey, Samuel, "Archimedes, Infinitesimals and the Law of Continuity: On Leibniz's Fictionalism”, en: Goldenbaum, Ursula y Jesseph, Douglas (eds.), Infinitesimal Differences: Controversies between Leibniz and his Contemporaries, Berlin \& New York, Walter de Gruyter, 2008, pp. 107-133.

Lützen, Jesper, "17th century arguments for the impossibility of the indefinite and the definite circle quadrature", Revue d'Histoire des Mathématiques, 20, 2014, pp. 211-251.

Malet, Antoni y Panza, Marco, "Wallis on Indivisibles", en Jullien, Vincent (ed.), Seventeenth-Century Indivisibles Revisited, Dordrecht, Birkäuser, 2015, pp. 307-346. 
Metius, Adriaan, Aritbmetica et geometric practica, Franeker, Rombertus Doyema, 1611.

Pascal, Blaise, Traité du triangle arithmétique, avec quelques autres petits traitez, sur la mesme matiere, París, G. Desprez, 1665.

Probst, Siegmund, "The Relation between Leibniz and Wallis: an Overview from New Sources and Studies", Quaderns d'Història de l'Enginyeria, XVI, 2018, pp. 189-208.

Raffo Quintana, Federico, "Leibniz on the requisites of an exact arithmetical quadrature", Studies in History and Philosophy of Science, 67, 2018, pp. 65-73.

Snell, Willebrord, Cyclometricus, De circuli dimensione secundum Logistarum abacos, \& ad Mechanicem accuratisima; atque omnium parabilissima, Leiden, ex officina Elzeviriana, 1621.

Van Ceulen, Ludolph, Vanden Circkel, Delft, Jan Andriesz Boeckvercooper, 1596.

Wallis, John, Arithmetica infinitorum, Oxford, 1656.

Wallis, John, Mechanica sive de motu tractatus geometricus. Oxford, Typis Guilielmi Godbid, 1670.

Wallis, John, The arithmetics of infinitesimals, traducción e introducción de Jacqueline A. Stedall, New York, Springer, 2004. 